\title{
Nanostructured Materials for Electrochemical Applications
}

\author{
Dina Fattakhova-Rohlfing \\ Ludwig-Maximilians-University Munchen, Germany \\ dina.fattakhova@cup.uni-muenchen.de
}

For the development of novel energy conversion and energy storage systems, both the choice of materials and their morphology are of great importance. Nanostructuring has a profound effect on the material's properties and is considered as one of the key routes towards the improvement of their efficiency. My research interests are focused on the development of nanostructured electrode materials for electrochemical, photoelectrochemical and photovoltaic applications, as well as understanding and controlling the processes influencing charge transfer and charge transport properties of the nanoscaled materials. In particular we work on the fabrication of transparent conducting electrodes with various types and dimensions of 3D-nanostructures acting as novel conducting platforms for immobilization of biological redox entities [1-5]. Furthermore we explore the potential of ultrasmall nanocrystals of transition metal oxides. We have developed a novel synthesis route giving an access to the smallest ever reported crystalline metal oxide nanoparticles with tunable composition and tunable electric, optical and electrochemical properties. Besides the reduced crystal size and increased interface resulting in enhanced charge transfer and shortened ion/electron diffusion pathways, chemical synthesis of nanomaterials often leads to metastable and non-stoichiometric phases due to kinetic rather than thermodynamic control of their formation, which turns out to be advantageous for electrocatalysis and for electrochemical energy storage. The developed nanoparticles and their assemblies into porous continuous networks demonstrate excellent performance as catalysts and co-catalysts for electrochemical water splitting [6-8], energy storage $[9,10]$ and in dye-sensitized solar cells $[11,12]$.

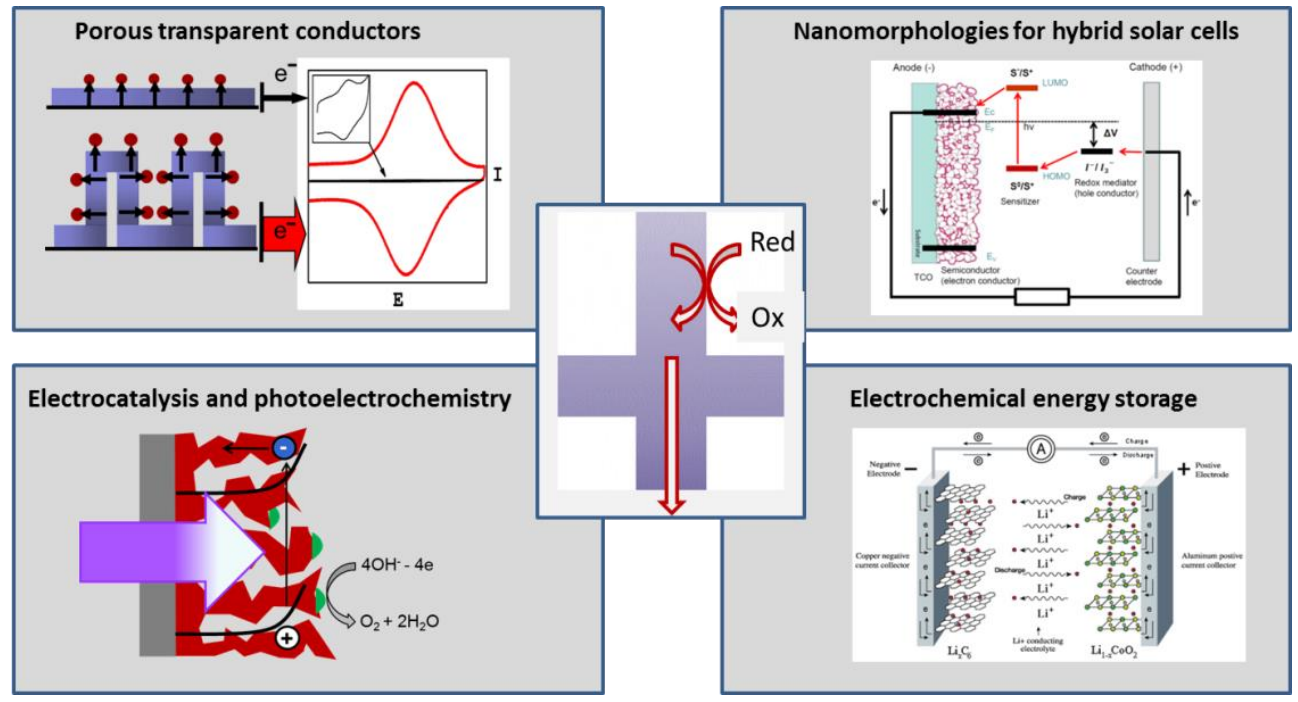

\section{References}

[1] V. Müller et al., Chem. Mater., vol. 21, pp. 5229, 2009.

[2] V. Müller et al., Small, vol. 6, pp. 633, 2010.

[3] K. Peters et al., Chem. Mater., vol. 27, pp. 1090, 2015.

[4] Y. Liu et al., ACS Nano, vol. 4, pp. 5373, 2010.

[5] Y. Liu et al, Chem. Sci., vol. 3, pp. 2367, 2012.

[6] K. Fominykh et al, Adv. Funct. Mater., vol. 24, pp. 3123, 2014.

[7] K. Fominykh et al, ACS nano, vol. 9, pp. 5180, 2015. 
[8] J. M. Feckl et al. (2015). Adv. Mater. Interfaces [Online]. DOI: 10.1002/admi.201500358;

[9] J. M. Szeifert et al, J. Am. Chem. Soc., vol. 132, pp. 12605, 2010.

[10] J. M. Feckl et al., Angew. Chem. Int. Ed., vol. 51, pp. 7459, 2012.

[11] J. M. Szeifert et al., Chem. Mater., vol. 24, pp. 659, 2012.

[12] J. M. Szeifert et al., Chem. Mater., vol. 21, pp. 1260, 2009. 